\title{
FAST EVALUATION OF EPHEMERIDES BY POLYNOMIAL APPROXIMATION IN THE CHEBYSHEV NORM
}

\author{
J. C. COMA, M. LARA AND T. J. LÓPEZ MORATALLA \\ Real Instituto y Observatorio de la Armada \\ 11110 San Fernando. Spain
}

Normally the planetary and satellite ephemerides are provided in tabular form, where the user interpolates between points in order to obtain the ephemerides. There are other methods of providing ephemerides by means of polynomial representations. The user is supplied with the coefficients of a set of polynomials which allow him a fast ephemerides evaluation.

In relation to Astronomy polynomial approximations are usually computed from a least squares fit. The disadvantage of this method is the absence of an estimate for the error: the magnitude of the error grows from the center of the interval towards the ends.

Another method no so frequently used is the uniform approximation in the Chebyshev norm. With this polynomial approximation, contrary to least squares, we obtain an uniform approximation of the function: the maxima and minima absolute values of the errors derived from this approximation are equal along the complete considered interval.

Fitting analytical functions, the method is well known. For instance, since 1980 the Bureau des Longitudes in their "Connaissance des Temps" computes series expansions in Chebyshev polynomials which, for certain intervals, represents their analytical theories.

The topic of this contribution is different. We use the uniform approximation in the Chebyshev norm in order to fit a data base. For the theory and details on the programming the reader is addressed to the references.

\section{Uniform approximation and "Almanaque Náutico"}

The "Real Instituto y Observatorio de la Armada en San Fernando" is editing the Almanaque Náutico continuously since 1792. Nowadays we use the data base DE200/LE200 of the Jet Propulsion Laboratory to compute our almanac. From basic ephemerides we select the desired data and then 
Polynomial degree and error estimation. Period 35 days.

\begin{tabular}{|c|c|c|c|c|}
\hline Body & $\begin{array}{c}\text { R.A. } \\
\varepsilon<10^{-5} \text { rad. }\end{array}$ & $\begin{array}{c}\delta \\
\varepsilon<10^{-5} \mathrm{rad} .\end{array}$ & $\begin{array}{c}r \\
\varepsilon<10^{-6} \mathrm{AU}\end{array}$ & $\begin{array}{c}V \\
\varepsilon<10^{-2} \\
\end{array}$ \\
\hline Sun & 4 & 4 & 4 & \\
\hline Moon & 24 & 21 & 7 & \\
\hline Venus & 6 & 7 & & 3 \\
\hline Mars & 5 & 4 & & 2 \\
\hline Jupiter & 4 & 3 & & 2 \\
\hline Saturn & 3 & 3 & & 2 \\
\hline
\end{tabular}

RA: Right ascension, $\delta$ : declination, $r$ : distance, $V$ : visual magnitude.

TABLE 1. Approximation polynomials for Almanaque Náutico.

we use uniform approximation in order to compress the data: taking an interval of 35 days and establishing a bound for the error of $10^{-5}$ radian, about 0.03 minutes of arc, we compute the planetary ephemerides using low degree polynomials except for the Moon. The necessary degrees are listed in Table 1.

The approximation polynomials provide a fast and simple way to compute the quantities that appear in the Almanaque Náutico and this method has been implemented as a part of the navigation system of the Spanish Navy.

\section{Acknowledgments}

We are indebt with Dr. Deprit who suggested us the application of the minimax method. The first author (J. C. C.) thanks Grupo de Mecánica Espacial in the University of Zaragoza where he developed the capital part of this work. This work has been supported in part by the Spanish Ministry of Education (DGICYT Project \# PB93-1236-C02-02) and by the Département de Mathématiques Spatiales at the CNES (Toulouse).

\section{References}

1. Barrodale, C.P.:1975, ACM Trans. Math. Software, 1, 264-270.

2. Deprit, A. and Picard, H.:1979, Naval Research Laboratory Report 8280.

3. Remez, E.:1957, General Computation Methods for Chebyshev Approximation, Izdat. Akad. Nauk. Ukranisk SSR, Kiev.

4. Schmitt, H.:1971, Discrete Chebyshev curve fit, Comm. ACM 14, 355-357.

5. Stiefel, E.L.:1958, Numerical Methods of Chebyshev Approximation, on Numerical Approximation, R. Langer (Ed.), U. Winsconsin, pp. 217-232.

6. Valleé-Poussin, C. de la.:1919, Leçons sur l'approximation des fonctions d'une variable réelle, Paris Gauthier-Villars, p. 75. 